\title{
Prostate-specific membrane antigen (PSMA) imaging: the past is prologue and the future is scintillating
}

\author{
Steven P. Rowe ${ }^{1,2}$, Michael A. Gorin ${ }^{1,2}$, Martin G. Pomper ${ }^{1,2}$ \\ ${ }^{1}$ The Russell H. Morgan Department of Radiology and Radiological Science, ${ }^{2}$ The James Buchanan Brady Urological Institute and Department of \\ Urology, Johns Hopkins University School of Medicine, Baltimore, MD, USA \\ Correspondence to: Steven P. Rowe, MD, PhD. Division of Nuclear Medicine and Molecular Imaging, The Russell H. Morgan Department of \\ Radiology and Radiological Science, Johns Hopkins University School of Medicine, 601 N. Caroline St., JHOC Room 3233, Baltimore, MD 21287, \\ USA. Email: srowe8@jhmi.edu. \\ Provenance and Peer Review: This article is commissioned and reviewed by the Section Editor Dr. Xiao Li (Department of Urology, Jiangsu Cancer \\ Hospital, Jiangsu Institute of Cancer Research, Nanjing Medical University Affiliated Cancer Hospital, Nanjing, China). \\ Response to: Choyke PL, Bouchelouche K. Prostate specific membrane antigen (PSMA) imaging: the past is prologue. Transl Androl Urol 2019;8:283-5.
}

Submitted Dec 22, 2019. Accepted for publication Jan 17, 2020.

doi: $10.21037 /$ tau. 2020.01 .27

View this article at: http://dx.doi.org/10.21037/tau.2020.01.27

A recent commentary by Choyke and Bouchelouche (1) highlighted a number of important points relevant to the origins and history of small-molecule inhibitors of prostate-specific membrane antigen (PSMA) and their use as imaging agents for positron emission tomography (PET). As the authors note, there has been rapid adoption of PSMA-targeted PET throughout much of the world (1), with hundreds of publications being generated each year (2). The facile incorporation of PSMA-targeted PET into clinical practice belies the tremendous amount of time and effort spent on medicinal chemistry to develop this new class of radiotracers, as well as the preclinical evaluations that were necessary to validate PSMA as a viable target for imaging prostate cancer $(3,4)$. Furthermore, as Choyke and Bouchelouche note in their manuscript, much of the early literature with PSMA-targeted PET was retrospective and/ or anecdotal (1), effectively putting the cart before the horse in regards to clinical validation (5), and potentially limiting future prospects for rigorous, randomized trials in some clinical applications (1).

Nonetheless, exciting developments continue to come to the forefront with PSMA, and there is every reason to believe that we have only scratched the surface. For example, prospective, multi-center studies in the United States (6) and ClinicalTrials.gov (NCT02981368 and NCT03739684) have positioned PSMA-targeted PET for regulatory approval in that jurisdiction in the near future. Such approval may be the impetus needed to continue the types of large, collaborative studies that will definitively elucidate the clinical utility of this new imaging technique in clinical scenarios such as pre-operative staging and biochemical recurrence. Beyond the sensitivity and specificity data from such studies, the more subtle prognostic information associated with scan findings should be sought. There are already indications that PSMA-targeted PET scans contain imaging biomarkers that are not accounted for simply by the detection efficiency of the scan (7), and this observation will need to be more thoroughly understood by leveraging the larger datasets that will continue to become available.

Dovetailing with the emerging data from multi-center studies is the worldwide experience that has led to an understanding of potential false-positive and false-negative findings as well as the appearances of numerous nonprostate cancer entities on PSMA-targeted PET. Indeed, the pitfalls that can lead to inaccurate staging of patients who are imaged with PSMA-targeted PET have been extensively described (8). This, in turn, has allowed for the development of standardized reporting systems [for example, (9)] that can reflect the inherent uncertainty that arises with normal variants, lesions with indeterminate levels of uptake, and other confusing imaging patterns. In our clinical practice, we make use of the PSMA reporting and data system (PSMA-RADS), which is structured as a 5 -point scale based on the likelihood of the presence of 
prostate cancer on a scan or in a specific individual lesion (9). PSMA-RADS and other structured reporting systems lay the groundwork for effective communication between interpreting image specialists and referring clinicians, which is of tremendous importance in an era when focal therapy options for patients with biochemically-recurrent or oligometastatic prostate cancer are often inferred from PSMA-targeted PET scan findings (2). More generally, we can expect that longer-term outcomes data from patients with low-volume advanced disease who receive focal therapy based on PSMA-targeted PET scan results will soon begin to become available, and whether such treatment decisions are appropriate will become more apparent.

Further, the expression of PSMA on the neovasculature of many non-prostate solid tumors has opened up the possibility of utilizing PSMA-targeted PET as a more generalizable cancer imaging modality (10). The literature on the applicability of PSMA-targeted radiotracers to the PET imaging of non-prostate cancers has been particularly focused on case reports, with a relatively limited number of larger and/or prospective studies (10). The bias associated with this pattern of publication almost certainly overestimates the clinical utility of PSMA-targeted PET in many cancers, emphasizing the need for further research on this topic. Beyond metrics such as the sensitivity and specificity for lesion detection, PSMA-targeted PET of non-prostate cancers may open up possibilities for treating patients harboring such malignancies with PSMA-based endoradiotherapy. Similarly, PSMA-targeted PET may serve as a non-invasive read-out of neovascular density and allow for the selection of patients that might benefit from neovascular-targeted therapies.

Lastly, just as with other imaging modalities, our ability to interpret PSMA-targeted PET is likely to be radically transformed by artificial intelligence (AI)/machine learning (ML). Initially, AI/ML will aid in lesion identification, segmentation, and categorization. However, those are likely to be only the first steps towards a more comprehensive role of AI/ML in PSMA-targeted PET imaging. Subsequent steps of $\mathrm{AI} / \mathrm{ML}$ incorporation into clinical practice may include deducing prognostic information, such as progression-free survival, as well as the selection of future therapies, based on a combination of PSMA-targeted PET scan findings and clinical information. Outside of the few prospective, multi-center studies that have been carried out, the tremendous number of scans that have been acquired as part of clinical routine in many parts of the world can already provide the basis for training AI/ML algorithms, potentially allowing for a relatively rapid adoption of these methods into clinical practice.

We thank Drs. Choyke and Bouchelouche for their excellent commentary that provided an overview of many of the important details regarding the timeline of the development of viable small-molecule radiotracers for imaging PSMA (1). We hope that the current manuscript continues this worthwhile discussion, only with a dedicated emphasis on the emerging and future applications of PSMA-targeted PET.

\section{Acknowledgments}

Funding: All authors receive research funding from Progenics Pharmaceuticals Inc.

\section{Footnote}

Conflicts of Interest: MG Pomper is a co-inventor on a US patent covering ${ }^{18} \mathrm{~F}$-DCFPyL and as such is entitled to a portion of any licensing fees and royalties generated by this technology. This arrangement has been reviewed and approved by the Johns Hopkins University in accordance with its conflict of interest policies. MA Gorin has served as a consultant for Progenics Pharmaceuticals Inc., the licensee of ${ }^{18} \mathrm{~F}$-DCFPyL. SP Rowe is a consultant for Progenics Pharmaceuticals Inc.

Ethical Statement: The authors are accountable for all aspects of the work in ensuring that questions related to the accuracy or integrity of any part of the work are appropriately investigated and resolved.

Open Access Statement: This is an Open Access article distributed in accordance with the Creative Commons Attribution-NonCommercial-NoDerivs 4.0 International License (CC BY-NC-ND 4.0), which permits the noncommercial replication and distribution of the article with the strict proviso that no changes or edits are made and the original work is properly cited (including links to both the formal publication through the relevant DOI and the license). See: https://creativecommons.org/licenses/by-ncnd/4.0\%.

\section{References}

1. Choyke PL, Bouchelouche K. Prostate specific membrane antigen (PSMA) imaging: the past is prologue. Transl 
Androl Urol 2019;8:283-5.

2. Murphy DG, Sweeney CJ, Tombal B. "Gotta catch 'em all”, or do we? Pokemet approach to metastatic prostate cancer. Eur Urol 2017;72:1-3.

3. Kiess AP, Banerjee SR, Mease RC, et al. Prostate-specific membrane antigen as a target for cancer imaging and therapy. Q J Nucl Med Mol Imaging 2015;59:241-68.

4. Rowe SP, Gorin MA, Pomper MG. Imaging of prostatespecific membrane antigen with small-molecule PET radiotracers: from the bench to advanced clinical applications. Annu Rev Med 2019;70:461-77.

5. Vapiwala N, Hofman MS, Murphy DG, et al. Strategies for evaluation of novel imaging in prostate cancer: putting the horse back before the cart. J Clin Oncol 2019;37:765-9.

6. Fendler WP, Calais J, Eiber M, et al. Assessment of $68 \mathrm{Ga}-$ PSMA-11 PET accuracy in localizing recurrent prostate cancer: a prospective single-arm clinical trial. JAMA Oncol 2019;5:856-63.

Cite this article as: Rowe SP, Gorin MA, Pomper MG. Prostate-specific membrane antigen (PSMA) imaging: the past is prologue and the future is scintillating. Transl Androl Urol 2020;9(2):840-842. doi: 10.21037/tau.2020.01.27
7. van Leeuwen PJ, Donswijk M, Nandurkar R, et al. Gallium-68-prostate-specific membrane antigen (68GaPSMA) positron emission tomography (PET)/computed tomography (CT) predicts complete biochemical response from radical prostatectomy and lymph node dissection in intermediate- and high-risk prostate cancer. BJU Int 2019;124:62-8.

8. Sheikhbahaei S, Afshar-Oromieh A, Eiber M, et al. Pearls and pitfalls in clinical interpretation of prostate-specific membrane antigen (PSMA)-targeted PET imaging. Eur J Nucl Med Mol Imaging 2017;44:2117-36.

9. Rowe SP, Pienta KJ, Pomper MG, et al. Proposal for a structured reporting system for prostate-specific membrane antigen-targeted PET imaging: PSMA-RADS version 1.0. J Nucl Med 2018;59:479-85.

10. Salas Fragomeni RA, Amir T, Sheikhbahaei S, et al. Imaging of nonprostate cancers using PSMA-targeted radiotracers: rationale, current state of the field, and a call to arms. J Nucl Med 2018;59:871-7. 